Research Article

\title{
New Existence Results for Nonlinear Fractional Integrodifferential Equations
}

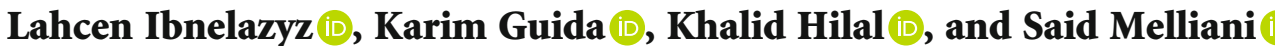

Sultan Moulay Slimane University, BP 523, 23000 Beni Mellal, Morocco

Correspondence should be addressed to Lahcen Ibnelazyz; ibnelazyzlahcen@gmail.com

Received 13 January 2021; Revised 23 February 2021; Accepted 3 March 2021; Published 31 March 2021

Academic Editor: Soheil Salahshour

Copyright (c) 2021 Lahcen Ibnelazyz et al. This is an open access article distributed under the Creative Commons Attribution License, which permits unrestricted use, distribution, and reproduction in any medium, provided the original work is properly cited.

This paper discusses a boundary value problem of nonlinear fractional integrodifferential equations of order $1<\alpha \leq 2$ and $1<\beta \leq 2$ and boundary conditions of the form $x(0)=x(1)={ }^{c} D^{\beta} x(1)={ }^{c} D^{\beta} x(0)=0$. Some new existence and uniqueness results are proposed by using the fixed point theory. In particular, we make use of the Banach contraction mapping principle and Krasnoselskii's fixed point theorem under some weak conditions. Moreover, two illustrative examples are studied to support the results.

\section{Introduction}

Fractional differential equations are relevant in many fields of science, such as chemistry, fluid systems, and electromagnetic; for more details about the theory of fractional differential equations and their applications, we invite the readers to see $[1-16]$ and the references therein.

Some physical applications of fractional differential equations include viscoelasticity, Schrodinger equation, fractional diffusion equation, and fractional relaxation equation; for more details, we refer the readers to [17].

In addition, fractional integrodifferential equations are used as an important tool to gain insight into some emerging problems from several science areas, for more details, we give the following references [18-23].

More recently, in [24], the existence and uniqueness of positive solutions for the fractional integrodifferential equation were proved.

In [25], the authors discussed the existence and uniqueness of solutions for nonlinear integrodifferential equations of fractional order with three-point nonlocal fractional boundary conditions. The existence of solutions for nonlinear fractional integrodifferential equations has been studied in [26].
Motivated by all these works and by the fact that there are no papers dealing with the new existence results for nonlinear fractional integrodifferential equations, in this work, we consider the existence and uniqueness of solutions for the following problem:

$$
\left\{\begin{array}{l}
{ }^{c} D^{\alpha}\left({ }^{c} D^{\beta}\right) x(t)=f(t, x(t), \Phi x(t), \psi x(t)), \quad t \in[0,1], \\
x(0)=x(1)={ }^{c} D^{\beta} x(1)={ }^{c} D^{\beta} x(0)=0,
\end{array}\right.
$$

where $1<\alpha \leq 2,1<\beta \leq 2,{ }^{c} D^{\alpha},{ }^{c} D^{\beta}$ are the Caputo fractional derivatives, $f:[0,1] \times \mathbb{R}^{3} \longrightarrow \mathbb{R}$ is a continuous function, and

$$
\begin{aligned}
& \Phi x(t)=\int_{0}^{t} \lambda(t, s) x(s) d s, \\
& \psi x(t)=\int_{0}^{t} \delta(t, s) x(s) d s,
\end{aligned}
$$

where $\lambda, \delta:[0,1] \times[0,1]-\longrightarrow[0,+\infty)$, with $\phi^{*}=\sup _{t \in[0,1]}$

$\int_{0}^{t} \lambda(t, s) d s\left|<\infty, \psi^{*}=\sup _{t \in[0,1]}\right| \int_{0}^{t} \delta(t, s) d s \mid<\infty$. 
This paper is organized as follows. In Section 2, we present some preliminaries and notations that will be required for the later sections. After that, in Section 3, we establish the main results by using the fixed point theory. And, in the last section, we give two examples to illustrate the results.

\section{Preliminaries and Notations}

In this section, we give some notations, definitions, and lemmas which will be required for the rest of the paper.

Definition 1 [5]. The fractional integral of order $\alpha>0$ with the lower limit zero for a function $f$ can be defined as

$$
I^{\alpha} f(t)=\frac{1}{\Gamma(\alpha)} \int_{0}^{t}(t-s)^{\alpha-1} f(s) d s
$$

Definition 2 [5]. The Caputo derivative of order $\alpha>0$ with the lower limit zero for a function $f$ can be defined as

$$
{ }^{c} D^{\alpha} f(t)=\frac{1}{\Gamma(n-\alpha)} \int_{0}^{t}(t-s)^{n-\alpha-1} f^{(n)}(s) d s,
$$

where $n \in \mathbb{N}, 0 \leq n-1<\alpha<n, t>0$.

Theorem 3 [27]. Let $M$ be a bounded, closed, convex, and nonempty subset of a Banach space X. Let $A$ and $B$ be two operators such that

(i) $A x+B y \in M$ whenever $x, y \in M$

(ii) $A$ is compact and continuous

(iii) $B$ is a contraction mapping

Then, there exists $z \in M$ such that $z=A z+B z$.

Lemma 4 [5]. Let $\alpha, \beta \geq 0$; then, the following relation hold:

$$
I^{\alpha} t^{\beta}=\frac{\Gamma(\beta+1)}{\Gamma(\alpha+\beta+1)} t^{\alpha+\beta} .
$$

Lemma 5 [5]. Let $n \in \mathbb{N}$ and $n-1<\alpha<n$. If $f$ is a continuous function, then we have

$$
I^{\alpha c} D^{\alpha} f(t)=f(t)+a_{0}+a_{1} t+a_{2} t^{2}+\cdots+a_{n-1} t^{n-1} .
$$

Lemma 6. Let $h \in C([0,1], \mathbb{R})$. Then, the unique solution of the problem

$$
\left\{\begin{array}{l}
{ }^{c} D^{\alpha}\left({ }^{c} D^{\beta}\right) x(t)=h(t), \quad t \in[0,1], \\
x(0)=x(1)={ }^{c} D^{\beta} x(1)={ }^{c} D^{\beta} x(0)=0,
\end{array}\right.
$$

is given by

$$
\begin{aligned}
x(t)= & \frac{1}{\Gamma(\alpha+\beta)} \int_{0}^{t}(t-s)^{\alpha+\beta-1} h(s) d s \\
& -\frac{t^{\beta+1}+t}{\Gamma(\alpha) \Gamma(\beta+2)} \int_{0}^{1}(1-s)^{\alpha-1} \times h(s) d s \\
& -\frac{t}{\Gamma(\alpha+\beta)} \int_{0}^{1}(1-s)^{\alpha+\beta-1} h(s) d s .
\end{aligned}
$$

Proof. By applying Lemma 5, we have

$$
\begin{aligned}
{ }^{c} D^{\beta} x(t) & =I^{\alpha} h(t)+a_{0}+a_{1} t, \\
x(t) & =I^{\alpha+\beta} h(t)+I^{\beta} a_{0}+I^{\beta} a_{1} t+a_{2}+a_{3} t,
\end{aligned}
$$

where $a_{0}, a_{1}, a_{2}, a_{3} \in \mathbb{R}$. So

$$
\begin{aligned}
x(t)= & \frac{1}{\Gamma(\alpha+\beta)} \int_{0}^{t}(t-s)^{\alpha+\beta-1} h(s) d s+\frac{t^{\beta}}{\Gamma(\beta+1)} a_{0} \\
& +\frac{t^{\beta+1}}{\Gamma(\beta+2)} a_{1}+a_{2}+a_{3} t .
\end{aligned}
$$

And by using ${ }^{c} D^{\beta} x(0)=x(0)=0$, we obtain $a_{0}=a_{2}=0$. As a result of ${ }^{c} D^{\beta} x(1)=0$, we have that

$$
a_{1}=-\frac{1}{\Gamma(\alpha)} \int_{0}^{1}(1-s)^{\alpha-1} h(s) d s .
$$

Now, we use $x(1)=0$ to get

$$
\begin{aligned}
a_{3}= & -\frac{1}{\Gamma(\alpha+\beta)} \int_{0}^{1}(1-s)^{\alpha+\beta-1} h(s) d s \\
& +\frac{1}{\Gamma(\alpha) \Gamma(\beta+2)} \int_{0}^{1}(1-s)^{\alpha-1} h(s) d s .
\end{aligned}
$$

By substituting the value of $a_{0}, a_{1}, a_{2}, a_{3}$, we obtain the following

$$
\begin{aligned}
x(t)= & \frac{1}{\Gamma(\alpha+\beta)} \int_{0}^{t}(t-s)^{\alpha+\beta-1} h(s) d s \\
& -\frac{t^{\beta+1}-t}{\Gamma(\alpha) \Gamma(\beta+2)} \int_{0}^{1}(1-s)^{\alpha-1} \times h(s) d s \\
& -\frac{t}{\Gamma(\alpha+\beta)} \int_{0}^{1}(1-s)^{\alpha+\beta-1} h(s) d s .
\end{aligned}
$$

Conversely, by direct computations, we obtain the desired result.

\section{Main Results}

Let $X$ be the Banach space of all continuous functions from $[0,1] \longrightarrow \mathbb{R}$ endowed with the norms $\|y\|=\sup \{|y(t)|: t$ $\in[0,1]\}$ and $\|y\|_{v}=\sup _{t \in[0,1]}\left(|y(t)| / e^{v t}\right)$, where $v>\left(\left(1+\phi^{*}+\right.\right.$ $\left.\left.\varphi^{*}\right) /(\Gamma(\alpha+\beta))\right)\|\sigma\|$, and $\sigma$ will be defined later. 


\section{Theorem 7. Assume that}

$\left(H_{1}\right)$ for all $t \in[0,1]$ and $x_{1}, x_{2}, x_{3}, y_{1}, y_{2}, y_{3} \in \mathbb{R}$, we have $\left|f\left(t, x_{1}, x_{2}, x_{3}\right)-f\left(t, y_{1}, y_{2}, y_{3}\right)\right| \leq \sigma(t)\left(\left|x_{1}-y_{1}\right|+\left|x_{2}-y_{2}\right|+\right.$ $\left.\left|x_{3}-y_{3}\right|\right)$ with $\sigma \in C([0,1] ;[0, \infty))$

$\left(H_{2}\right)|f(t, x, y, z)| \leq \theta(t), \forall(t, x, y, z) \in[0,1] \times \mathbb{R}^{3}$ with $\theta$ $\in C\left([0,1] ; \mathbb{R}^{+}\right)$. Then, the problem (1) has at least one solution.

Proof. We consider the ball $B_{r}=\left\{y \in X:\|y\|_{v} \leq r\right\}$ with $r \geq$ $(\|\theta\| / v)\left(\left(\left(2\left(e^{v}-1\right)\right) /(\Gamma(\alpha) \Gamma(\beta+2))\right)+\left(\left(e^{v}\right) /(\Gamma(\alpha+\beta))\right)\right)$

.We define the operators $F=F_{1}+F_{2}$ on $B_{r}$, where.

$$
F_{1 y(t)}=\frac{1}{\Gamma(\alpha+\beta)} \int_{0}^{t}(t-s)^{\alpha+\beta-1} f(s, y(s), \phi y(s), \psi y(s)) d s
$$

$$
\begin{aligned}
F_{2 y(t)}= & -\frac{t^{\beta+1}-t}{\Gamma(\alpha) \Gamma(\beta+2)} \int_{0}^{1}(1-s)^{\alpha-1} f(s, y(s), \phi y(s), \psi y(s)) d s \\
& -\frac{1}{\Gamma(\alpha+\beta)} \int_{0}^{1}(1-s)^{\alpha+\beta-1} f(s, y(s), \phi y(s), \psi y(s)) d s .
\end{aligned}
$$

For $x, y \in B_{r}$, we have

$$
\begin{aligned}
& \left\|F_{1 x(t)}\right\|_{v} \leq \sup _{t \in[0,1]} \frac{1}{e^{v t}}\left|\frac{1}{\Gamma(\alpha+\beta)} \int_{0}^{t}(t-s)^{\alpha+\beta-1} f(s, x(s), \phi x(s), \psi x(s)) d s\right| \\
& \leq \sup _{t \in[0,1]} \frac{1}{e^{v t}} \frac{1}{\Gamma(\alpha+\beta)} \int_{0}^{t}(t-s)^{\alpha+\beta-1}|\theta(s)| d s \\
& \leq \sup _{t \in[0,1]} \frac{1}{e^{v t}} \frac{1}{\Gamma(\alpha+\beta)} \int_{0}^{t}(t-s)^{\alpha+\beta-1} \frac{|\theta(s)| e^{v s}}{e^{v s}} d s \\
& \leq \sup _{t \in[0,1]} \frac{\|\theta\|_{v}}{e^{v t} \Gamma(\alpha+\beta)} \int_{0}^{t}(t-s)^{\alpha+\beta-1} e^{v s} d s \\
& \leq \sup _{t \in[0,1]} \frac{\|\theta\|_{v}}{e^{v t} \Gamma(\alpha+\beta)} \int_{0}^{t} e^{v s} d s \leq \sup _{t \in[0,1]} \frac{\|\theta\|_{v}}{v \Gamma(\alpha+\beta)} \frac{e^{v t}-1}{e^{v t}} \\
& \leq \frac{\|\theta\|_{v}}{v \Gamma(\alpha+\beta)} \\
& \left\|F_{2 y(t)}\right\|_{v} \leq \sup _{t \in[0,1]} \frac{1}{e^{v t}} \mid-\frac{t^{\beta+1}-t}{\Gamma(\alpha) \Gamma(\beta+2)} \int_{0}^{1}(1-s)^{\alpha-1} f(s, y(s), \phi y(s), \psi y(s)) d s \\
& -\frac{t}{\Gamma(\alpha+\beta)} \int_{0}^{1}(1-s)^{\alpha+\beta-1} f(s, y(s), \phi y(s), \psi y(s)) d s \mid \\
& \leq \sup _{t \in[0,1]} \frac{1}{e^{v t}}\left[\frac{2}{\Gamma(\alpha) \Gamma(\beta+2)} \int_{0}^{1}(1-s)^{\alpha-1} \frac{|\theta(s)| e^{v s}}{e^{v s}} d s\right. \\
& \left.+\frac{1}{\Gamma(\alpha+\beta)} \times \int_{0}^{1}(1-s)^{\alpha+\beta-1} \frac{|\theta(s)| e^{v s}}{e^{v s}} d s\right] \\
& \leq \sup _{t \in[0,1]} \frac{\|\theta\|_{v}}{e^{v t}}\left[\frac{2}{\Gamma(\alpha) \Gamma(\beta+2)} \int_{0}^{1}(1-s)^{\alpha-1} e^{v s} d s+\frac{1}{\Gamma(\alpha+\beta)}\right. \\
& \left.\times \int_{0}^{1}(1-s)^{\alpha+\beta-1} e^{v s} d s\right] \leq \sup _{t \in[0,1]} \frac{\|\theta\|_{v}}{e^{v t}}\left[\frac{2}{\Gamma(\alpha) \Gamma(\beta+2)} \int_{0}^{1} e^{v s} d s\right. \\
& \left.+\frac{1}{\Gamma(\alpha+\beta)} \int_{0}^{1} e^{v s} d s\right] \leq \sup _{t \in[0,1]} \frac{\|\theta\|_{v}}{v e^{v t}}\left(\frac{2\left(e^{v}-1\right)}{\Gamma(\alpha) \Gamma(\beta+2)}+\frac{e^{v}-1}{\Gamma(\alpha+\beta)}\right) \\
& \leq \frac{\|\theta\|_{v}}{v}\left(\frac{2\left(e^{v}-1\right)}{\Gamma(\alpha) \Gamma(\beta+2)}+\frac{\left(e^{v}-1\right)}{\Gamma(\alpha+\beta)}\right) \text {. }
\end{aligned}
$$

Therefore,

$$
\left\|F_{1} x+F_{2} y\right\|_{v} \leq \frac{\|\theta\|}{v}\left(\frac{2\left(e^{v}-1\right)}{\Gamma(\alpha) \Gamma(\beta+2)}+\frac{e^{v}}{\Gamma(\alpha+\beta)}\right) .
$$

Then,

$$
F_{1} x+F_{2} y \in B_{r}
$$

Now, we prove that $F_{1}$ is a contraction. For $x, y \in B_{r}$, we have

$$
\begin{aligned}
& \left\|F_{1} y(t)-F_{1} x(t)\right\|_{v} \leq \sup _{t \in[0,1]} \frac{1}{\Gamma(\alpha+\beta) e^{v t}} \int_{0}^{t}(t-s)^{\alpha+\beta-1} \\
& \quad \times|f(s, y(s), \phi y(s), \psi y(s))-f(s, x(s), \phi x(s), \psi x(s))| d s \\
& \leq \sup _{t \in[0,1]} \frac{1}{\Gamma(\alpha+\beta) e^{v t}} \int_{0}^{t}(t-s)^{\alpha+\beta-1} \sigma(s)(|y(s)-x(s)| \\
& \quad+|\phi y(s)-\phi x(s)|+|\psi y(s)-\psi x(s)|) d s \leq \sup _{t \in[0,1]} \frac{\|\sigma\|}{\Gamma(\alpha+\beta) e^{v t}} \\
& \quad \cdot \int_{0}^{t} e^{v s}\left(\|y-x\|_{v}+\phi *\|y-x\|_{v}+\psi *\|y-x\|_{v}\right) d s \\
& \leq \sup _{t \in[0,1]} \frac{(1+\phi *+\psi *)\|\sigma\| e^{v t}-1}{v \Gamma(\alpha+\beta)}\|y-x\|_{v} \\
& \leq \frac{(1+\phi *+\psi *)\|\sigma\|\|y-x\|_{v}}{\Gamma(\alpha+\beta) v} .
\end{aligned}
$$

By using the condition of the new norm, we conclude that $F_{1}$ is a contraction.

Next, we will prove that $F_{2}$ is compact and continuous.

Continuity of $f$ implies that the operator $F_{2}$ is continuous. Also, $F_{2}$ is uniformly bounded on $B_{r}$ as

$$
\left\|F_{2} y\right\| \leq \frac{\|\theta\|_{v}\left(e^{v}-1\right)}{v}\left(\frac{2}{\Gamma(\alpha) \Gamma(\beta+2)}+\frac{1}{\Gamma(\alpha+\beta)}\right) .
$$

Suppose that $0 \leq t_{1}<t_{2} \leq 1$. We have

$$
\begin{aligned}
& \left|F_{2} y\left(t_{2}\right)-F_{2} y\left(t_{1}\right)\right| \leq \frac{\left|t_{2}^{\beta+1}-t_{1}^{\beta+1}\right|+\left|t_{2}-t_{1}\right|}{\Gamma(\alpha) \Gamma(\beta+2)} \int_{0}^{1}(1-s)^{\alpha-1} \\
& \times|f(s, x(s), \phi x(s), \psi x(s))| d s+\frac{\left|t_{2}-t_{1}\right|}{\Gamma(\alpha+\beta)} \\
& \cdot \int_{0}^{1}(1-s)^{\alpha+\beta-1}|f(s . x(s), \phi x(s), \psi x(s))| d s .
\end{aligned}
$$

Then, $\left|F_{2} y\left(t_{2}\right)-F_{2} y\left(t_{1}\right)\right| \longrightarrow 0$, as $t_{1} \longrightarrow t_{2}$ independently from $y \in B_{r}$.

This shows that the operator $F_{2}$ is relatively compact on $B_{r}$. Thus, by the Arzela Ascoli theorem, we obtain that $F_{2}$ is compact on $B_{r}$.

By the Krasnoselskii fixed point theorem, the problem (1) has at least one solution on $B_{r}$. 
Theorem 8. Suppose that $f:[0,1] \times \mathbb{R}^{3} \longrightarrow \mathbb{R}$ is a continuous function satisfying

$\left(H_{1}\right)$ for all $t \in[0,1]$ and $x_{1}, x_{2}, x_{3}, y_{1}, y_{2}, y_{3} \in \mathbb{R}$, we have $\left|f\left(t, x_{1}, x_{2}, x_{3}\right)-f\left(t, y_{1}, y_{2}, y_{3}\right)\right| \leq \sigma(t)\left(\left|x_{1}-y_{1}\right|+\left|x_{2}-y_{2}\right|+\right.$ $\left.\left|x_{3}-y_{3}\right|\right)$ with $\sigma(t) \in L^{1}([0,1] ;[0, \infty))$. Then, there exists a unique solution for the problem (1) under the following condition: $r_{1}<1$, where $r_{1}=2(1+\phi *+\psi *) \sigma *((1 / \Gamma(\alpha+\beta))+$ $(1 / \Gamma(\alpha) \Gamma(\beta+2)))$,

with $\sigma *=\int_{0}^{1} \sigma(t) d t$.

Proof. Define $F: X \longrightarrow X$ by

$$
\begin{aligned}
F x(t)= & \frac{1}{\Gamma(\alpha+\beta)} \int_{0}^{t}(t-s)^{\alpha+\beta-1} f(s, x(s), \phi x(s), \psi x(s), \psi x(s)) d s \\
& -\frac{t^{\beta+1}-t}{\Gamma(\alpha) \Gamma(\beta+2)} \int_{0}^{1}(1-s)^{\alpha-1} f(s, x(s), \phi x(s), \psi x(s)) d s \\
& -\frac{t}{\Gamma(\alpha+\beta)} \int_{0}^{1}(1-s)^{\alpha+\beta-1} f(s, x(s), \phi x(s), \psi x(s)) d s .
\end{aligned}
$$

Setting $\sup _{0 \leq t \leq 1}|f(t, 0,0,0)|=P$.

We consider the set $B_{r}=\{x \in X:\|x\| \leq r\}$, where $r \geq$ $\left(r_{2} /\left(1-r_{1}\right)\right)$, with

$$
r_{2}=2 P\left(\frac{1}{\Gamma(\alpha+\beta)}+\frac{1}{\Gamma(\alpha) \Gamma(\beta+2)}\right)
$$

For each $t \in[0,1]$ and $x \in B_{r}$, we have

$$
\begin{aligned}
|F x(t)| \leq & \frac{1}{\Gamma(\alpha+\beta)} \int_{0}^{t}(t-s)^{\alpha+\beta-1}|f(s, x(s), \phi x(s), \psi x(s))| d s \\
& +\frac{t^{\beta+1}+t}{\Gamma(\alpha) \Gamma(\beta+2)} \int_{0}^{1}(1-s)^{\alpha-1}|f(s, x(s), \phi x(s), \psi x(s))| d s \\
& +\frac{t}{\Gamma(\alpha+\beta)} \int_{0}^{1}(1-s)^{\alpha+\beta-1}|f(s, x(s), \phi x(s), \psi x(s))| \\
\leq & \frac{1}{\Gamma(\alpha+\beta)} \int_{0}^{1}(t-s)^{\alpha+\beta-1}(\mid f(s, x(s), \phi x(s), \psi x(s)) \\
& -f(s, 0,0,0)|+| f(s, 0,0,0) \mid) d s+\frac{t^{\beta+1}+t}{\Gamma(\alpha) \Gamma(\beta+2)} \\
& \cdot \int_{0}^{1}(1-s)^{\alpha-1} \times(|f(s, x(s), \phi x(s), \psi x(s))-f(s, 0,0,0)| \\
& +|f(s, 0,0,0)|) d s+\frac{1}{\Gamma(\alpha+\beta)} \\
& \cdot \int_{0}^{1}(t-s)^{\alpha+\beta-1}(|f(s, x(s), \phi x(s), \psi x(s))-f(s, 0,0,0)| \\
& +|f(s, 0,0,0)|) d s \leq \frac{1}{\Gamma(\alpha+\beta)} \\
& \cdot \int_{0}^{1}(t-s)^{\alpha+\beta-1}(\sigma(s)(|x(s)|+|\phi x(s)|+|\psi x(s)|)+P) d s \\
& +\frac{2}{\Gamma(\alpha) \Gamma(\beta+2)} \int_{0}^{1}(1-s)^{\alpha-1}(\sigma(s)(|x(s)|+|\phi x(s)| \\
& |\psi x(s)|)+P) d s+\frac{2}{\Gamma(\alpha+\beta)} \int_{0}^{1}(1-s)^{\alpha+\beta-1}(\sigma(s)(|x(s)| \\
& =1
\end{aligned}
$$

$$
\begin{aligned}
& +|\phi x(s)|+|\psi x(s)|)+P) d s \leq \frac{(1+\phi *+\psi *)\|x\|}{\Gamma(\alpha+\beta)} \\
& \quad \cdot \int_{0}^{1} \sigma(s) d s+\frac{P}{\Gamma(\alpha+\beta)} \int_{0}^{t}(t-s)^{\alpha+\beta-1} d s+\frac{2(1+\phi *+\psi *)\|x\|}{\Gamma(\alpha) \Gamma(\beta+2)} \\
& \quad \cdot \int_{0}^{1} \sigma(s) d s+\frac{2 P}{\Gamma(\alpha) \Gamma(\beta+2)}+\frac{(1+\phi *+\psi *)\|x\|}{\Gamma(\alpha+\beta)} \int_{0}^{1} \sigma(s) d s \\
& \quad+\frac{P}{\Gamma(\alpha+\beta)} \leq \frac{2(1+\phi *+\psi *)\|x\|}{\Gamma(\alpha+\beta)} \sigma *+\frac{2 P}{\Gamma(\alpha+\beta)} \\
& +\frac{2 P}{\Gamma(\alpha) \Gamma(\beta+2)}+\frac{2(1+\phi *+\psi *)\|x\|}{\Gamma(\alpha) \Gamma(\beta+2)} \sigma * \\
& \leq 2(1+\phi *+\psi *) \sigma *\left(\frac{1}{\Gamma(\alpha+\beta)}+\frac{1}{\Gamma(\alpha) \Gamma(\beta+2)}\right)\|x\| \\
& \quad+2 P\left(\frac{1}{\Gamma(\alpha+\beta)}+\frac{1}{\Gamma(\alpha) \Gamma(\beta+2)}\right) .
\end{aligned}
$$

Then, $\|F x\| \leq r$.

Therefore, $F B_{r} \subseteq B_{r}$.

Next, we show that $F$ is a contraction mapping.

For $x, y \in B_{r}$, we have

$|F x(t)-F y(t)| \leq \frac{1}{\Gamma(\alpha+\beta)} \int_{0}^{t}(t-s)^{\alpha+\beta-1} \mid f(s, x(s), \phi x(s), \psi x(s))$

$-f(s, y(s), \phi y(s), \psi y(s)) \mid d s+\frac{t^{\beta+1}+t}{\Gamma(\alpha) \Gamma(\beta+2)} \int_{0}^{t}(1-s)^{\alpha-1}$

$\times(|f(s, x(s), \phi x(s), \psi x(s))-f(s, y(s), \phi y(s), \psi y(s))|) d s$

$+\frac{1}{\Gamma(\alpha+\beta)} \int_{0}^{t}(1-s)^{\alpha+\beta-1}(\mid f(s, x(s), \phi x(s), \psi x(s))$

$-f(s, y(s), \phi y(s), \psi y(s)) \mid) d s \leq \frac{1}{\Gamma(\alpha+\beta)}$

$\cdot \int_{0}^{t}(t-s)^{\alpha+\beta-1} \sigma(s)(|x(s)-y(s)|+|\phi x(s)-\phi y(s)|$

$+|\psi x(s)-\psi y(s)|) d s+\frac{t^{\beta+1}+t}{\Gamma(\alpha) \Gamma(\beta+2)} \int_{0}^{t}(1-s)^{\alpha-1} \sigma(s)$

$\times(|x(s)-y(s)|+|\phi x(s)-\phi y(s)|+|\psi x(s)-\psi y(s)|) d s$

$+\frac{t}{\Gamma(\alpha+\beta)} \int_{0}^{1}(1-s)^{\alpha+\beta-1} \sigma(s)(|x(s)-y(s)|+|\phi x(s)-| \phi y(s)$

$+|\psi x(s)-\psi y(s)|) d s \leq \frac{(1+\phi *+\psi *)\|x-y\|}{\Gamma(\alpha+\beta)} \int_{0}^{1} \sigma(s) d s$

$+\frac{2(1+\phi *+\psi *)\|x-y\|}{\Gamma(\alpha) \Gamma(\beta+2)} \times \int_{0}^{1} \sigma(s) d s+\frac{(1+\phi *+\psi *)\|x-y\|}{\Gamma(\alpha+\beta)}$

$\cdot \int_{0}^{1} \sigma(s) d s+\frac{1}{\Gamma(\alpha+\beta)} \int_{0}^{1}(1-s)^{\alpha+\beta-1} \sigma(s)(|x(s)-y(s)|$

$+|\phi x(s)-\phi y(s)|+|\psi x(s)-\psi y(s)|) d s \leq \frac{(1+\phi *+\psi *) \| x-y||}{\Gamma(\alpha+\beta)}$

$\cdot \int_{0}^{1} \sigma(s) d s+\frac{2(1+\phi *+\psi *)\|x-y\|}{\Gamma(\alpha) \Gamma(\beta+2)} \times \int_{0}^{1} \sigma(s) d s$

$+\frac{(1+\phi *+\psi *)\|x-y\|}{\Gamma(\alpha+\beta)} \int_{0}^{1} \sigma(s) d s \leq \frac{2(1+\phi *+\psi *)\|x-y\|}{\Gamma(\alpha+\beta)} \sigma *$

$+\frac{2(1+\phi *+\psi *)\|x-y\|}{\Gamma(\alpha) \Gamma(\beta+2)} \sigma * \leq 2(1+\phi *+\psi *) \sigma *$

$\cdot\left(\frac{1}{\Gamma(\alpha+\beta)}+\frac{1}{\Gamma(\alpha) \Gamma(\beta+2)}\right)\|x-y\|$. 
Since $r_{1}<1$, then $F$ is a contraction. Therefore, the system (1) has a unique solution.

\section{Examples}

In this section, we give two examples to show the applicability of our results.

Example 1. Consider the following problem:

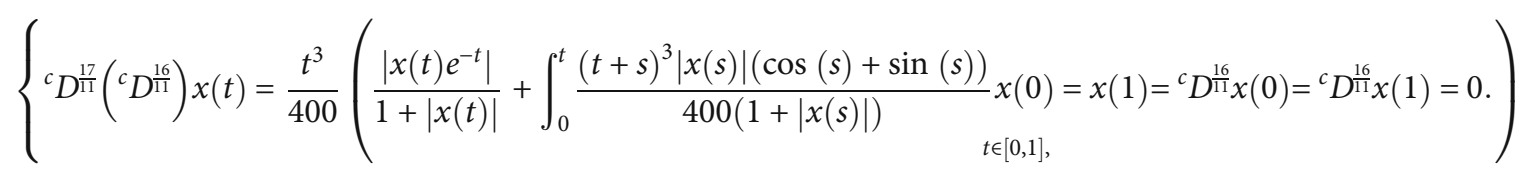

Here,

$$
\begin{aligned}
\beta & =\frac{16}{11}, \\
\alpha & =\frac{17}{11}, \\
f(t, x, y, z) & =\frac{t^{3}}{400}\left(\frac{|x(t)| e^{-t}}{1+|x(t)|}+\frac{|y(t)| \cos (t)}{1+|y(t)|}+\frac{|z(t)| \sin (t)}{1+|z(t)|}\right), \\
\lambda(t, s) & =\delta(t, s)=\frac{(t+s)^{3}}{400}, \\
\sigma(t) & =\frac{t^{3}}{400}, \\
\theta(t) & =\frac{3 t^{3}}{400} .
\end{aligned}
$$

It follows that

$$
\begin{aligned}
& \phi *=\psi *=\frac{15}{1600}, \\
& \sigma *=\frac{1}{1600} .
\end{aligned}
$$

Then, by Theorem 7, we obtain that the problem (26) has at least one solution.

Example 2. Consider the following system:

$$
\left\{\begin{array}{l}
{ }^{c} D^{10}\left({ }^{c} D^{\frac{11}{7}}\right) x(t)=\frac{t^{2}}{200}\left(\frac{1}{1+|x(t)|}+\frac{1}{100} \int_{0}^{t} t^{4} s^{3} x(s) d s\right), \quad t \in[0,1], \\
x(0)=x(1)={ }^{c} D^{\frac{11}{7}} x(0)={ }^{c} D^{\frac{11}{7}} x(1)=0 .
\end{array}\right.
$$

Here,

$$
\begin{aligned}
B & =\frac{11}{7}, \\
\alpha & =\frac{10}{7} \\
f(t, x, y, z) & =\frac{t^{2}}{200}\left(\frac{1}{1+|x(t)|}+y(t)+z(t)\right), \\
\lambda(t, s) & =\delta(t, s)=\frac{t^{4} s^{3}}{200}, \\
\sigma(t) & =\frac{t^{2}}{200} .
\end{aligned}
$$

It is clear that

$$
\begin{aligned}
& \phi *=\psi *=\frac{1}{800}, \\
& \sigma *=\frac{1}{600}, \\
& r_{1} \approx 0,0036 .
\end{aligned}
$$

By Theorem 8, we conclude that the problem (29) has a unique solution.

\section{Conclusion}

In this paper, we proved the existence and uniqueness of solutions for nonlinear fractional integrodifferential equations of order $1<\alpha \leq 2$ and $1<\beta \leq 2$ using the Banach contraction mapping principle and Krasnoselskii's fixed point theorem under some weak conditions. Furthermore, we provided two examples to illustrate the main results.

\section{Data Availability}

No data were used to support this study.

\section{Conflicts of Interest}

The authors declare that there are no conflicts of interest regarding the publication of this paper. 


\section{References}

[1] V. Lakshmikantham, "Theory of fractional functional differential equations," Nonlinear Anal, vol. 69, no. 10, pp. 3337-3343, 2008.

[2] V. Lakshmikantham and A. S. Vatsala, "Basic theory of fractional differential equations," Nonlinear Anal, vol. 69, no. 8, pp. 2677-2682, 2008.

[3] R. Hilfer, Applications of Fractional Calculus in Physics, World Scientific, Singapore, 2000.

[4] K. S. Miller and B. Ross, An Introduction to the Fractional Calculus and Fractional Differential Equations, Wiley, New York, 1993.

[5] I. Podlubny, Fractional Differential Equations, Academic Press, New York, 1993.

[6] Y. Zhou, Basic Theory of Fractional Differential Equations, Xiangtan University, China, 2014.

[7] A. A. Kilbas, H. M. Srivastava, and J. J. Trujillo, Theory and Applications of Fractional Differential Equations, NorthHolland Mathematics Studies, vol. 204, Elsevier, Amsterdam, 2006.

[8] K. Hilal, L. Ibnelazyz, K. Guida, and S. Melliani, Existence of Mild Solutions for an Impulsive Fractional IntegroDifferential Equations with Non-Local Condition, Springer Nature Switzerland AG, 2019.

[9] K. Hilal, K. Guida, L. Ibnelazyz, and M. Oukessou, Existence Results for an Impulsive Fractional Integro-Differential Equations with Non-Compact Semigroup, Springer Nature Switzerland AG, 2019.

[10] K. Guida, K. Hilal, L. Ibnelazyz, and M. Mei, "Existence of mild solutions for a class of impulsive Hilfer fractional coupled systems," Advances in Mathematical Physics, vol. 2020, Article ID 8406509, 12 pages, 2020.

[11] K. Hilal, L. Ibnelazyz, K. Guida, and S. Melliani, "Fractional Langevin equations with nonseparated integral boundary conditions," Advances in Mathematical Physics, vol. 2020, Article ID 3173764, 8 pages, 2020.

[12] K. Guida, K. Hilal, and L. Ibnelazyz, "Existence results for a class of coupled Hilfer fractional pantograph differential equations with nonlocal integral boundary value conditions," Advances in Mathematical Physics, vol. 2020, Article ID 8898292, 8 pages, 2020.

[13] L. Ibnelazyz, K. Guida, S. Melliani, and K. Hilal, “On a nonlocal multipoint and integral boundary value problem of nonlinear fractional integrodifferential equations," Journal of Function Spaces, vol. 2020, Article ID 8891736, 8 pages, 2020.

[14] L. Ibnelazyz, K. Guida, K. Hilal, and S. Melliani, "Existence results for nonlinear fractional integro-differential equations with integral and antiperiodic boundary conditions," Computational and Applied Mathematics, vol. 40, no. 1, article 33, 2021.

[15] M. I. el Bahi and K. Hilal, "Lie symmetry analysis, exact solutions, and conservation laws for the generalized timefractional KdV-like equation," Journal of Function Spaces, vol. 2021, Article ID 6628130, 10 pages, 2021.

[16] S. Muthaiah, D. Baleanu, and N. Gopal Thangaraj, "Existence and Hyers-Ulam type stability results for nonlinear coupled system of Caputo-Hadamard type fractional differential equations," Aims Mathematics, vol. 6, no. 1, pp. 168-194, 2021.
[17] M. Rahimy, "Applications of fractional differential equations," Applied Mathematical Sciences, vol. 4, no. 50, pp. 2453-2461, 2010.

[18] D. Baleanu, K. Ghafarnezhad, and S. Rezapour, "On a three step crisis integro-differential equation," Advances in Difference Equations, vol. 2019, no. 1, Article ID 153, 2019.

[19] D. Baleanu, K. Ghafarnezhad, S. Rezapour, and M. Shabibi, "On the existence of solutions of a three steps crisis integrodifferential, equation," Advances in Difference Equations, vol. 2018, no. 1, Article ID 135, 2018.

[20] B. Ahmad, S. K. Ntouyas, R. P. Agarwal, and A. Alsaedi, "Existence results for sequential fractional integro-differential equations with nonlocal multi-point and strip conditions," Boundary Value Problems, vol. 2016, no. 1, Article ID 205, 2016.

[21] B. Ahmad and S. Sivasundaram, "On four-point nonlocal boundary value problems of nonlinear integro- differential equations of fractional order," Applied Mathematics and Computation, vol. 217, no. 2, pp. 480-487, 2010.

[22] D. Baleanu, S. Z. Nazemi, and S. Rezapour, "Existence and uniqueness of solutions for multi-term nonlinear fractional integro-differential equations," Advances in Difference Equations, vol. 2013, no. 1, 2013.

[23] D. Baleanu, S. Etemad, and S. Rezapour, "On a fractional hybrid integro-differential equation with mixed hybrid integral boundary value conditions by using three operators," Alexandria Engineering Journal, vol. 59, no. 5, pp. 30193027, 2020.

[24] Y. Wang and L. Liu, "Uniqueness and existence of positive solutions for the fractional integro-differential equation," Boundary Value Problems, vol. 2017, no. 1, Article ID 12, 2017.

[25] A. Alsaedi and B. Ahmad, "Existence of solutions for nonlinear fractional integro-differential equations with three-point nonlocal fractional boundary conditions," Advances in Difference Equations, vol. 2010, no. 1, Article ID 691721, 2010.

[26] A. Bragdi, A. Frioui, and A. Guezane Lakoud, "Existence of solutions for nonlinear fractional integro-differential equations," Advances in Difference Equations, vol. 2020, no. 1, Article ID 418, 2020.

[27] A. Krasnoselskii, "Two remarks on the method of successive approximations," Uspekhi Matematicheskikh Nauk, vol. 10, pp. 123-127, 1955. 